\title{
X-RAY EMISSION AND REFLECTION IN AGNS
}

\author{
HAGAI NETZER \\ School of Physics and Astronomy and the Wise Observatory \\ Tel Aviv University
}

\begin{abstract}
X-ray spectra of many AGNs are dominated by strong absorption-like features at low (0.5-1 keV) energies. Recent progress in modeling AGNs indicate that pure X-ray absorption is unlikely to be observed and the contribution of emission and reflection must be significant. Several such models are shown and compared with what is likely to be observed by two X-ray experiments, ROSAT and $A S C A$. It is argued that $\mathrm{X}$-ray colors may be useful diagnostic tools in analyzing such spectra.
\end{abstract}

\section{Introduction}

Many low luminosity AGNs show evidence of X-ray absorption at around $1 \mathrm{keV}$ (e.g. Reichert et al. 1985) and recent observations (e.g. Weaver et al. 1993) hint to the presence of $\mathrm{X}$-ray emission lines at around $1-2 \mathrm{keV}$. The absorption has been interpreted as due to neutral ("cold") intrinsic absorber with partial covering (e.g. Reichert, Mushotzky \& Holt, 1986) or highly ionized ("warm") intrinsic absorber (e.g. Halpern, 1984; Krolik \& Kallman, 1984, Yaqoob, Warwick \& Pounds 1989). The observed strength of the features have been used to estimate the column density and level of ionization of the absorbing gas (the "warm absorber" case) or the covering factor of the cold material.

A recent work by Netzer (1993) discusses the physical conditions in the gas thought to give rise to the observed $\mathrm{X}$-ray absorption, and shows that emission and reflection by the highly ionized material is important in modifying the shape and the depth of the absorption features. Such effects are likely to show up in medium and high resolution $\mathrm{X}$-ray spectra.

This short contribution gives several examples of recently calculated X-ray spectra, demonstrates the importance of $\mathrm{X}$-ray emission and reflection, and suggests new ways of analyzing data by means of X-ray colors.

\section{X-ray Absorption, Emission and Reflection}

The strength of X-ray absorption features depend on the column density, level of ionization and the fractional obscuration of the source. X-ray emission and reflection are important at all situations and at most energies. The relative strength and equivalent width of such components is a function of the ionization parameter and the covering fraction.

The appearance of the spectrum depend on the origin of the X-ray reprocessing material. If these are clouds on the line of sight, then strong absorption features are likely to show up. If this is a warm material near the $\mathrm{X}$-ray source, such as a thin accretion disk, the spectrum is dominated by emission and reflection. Most 

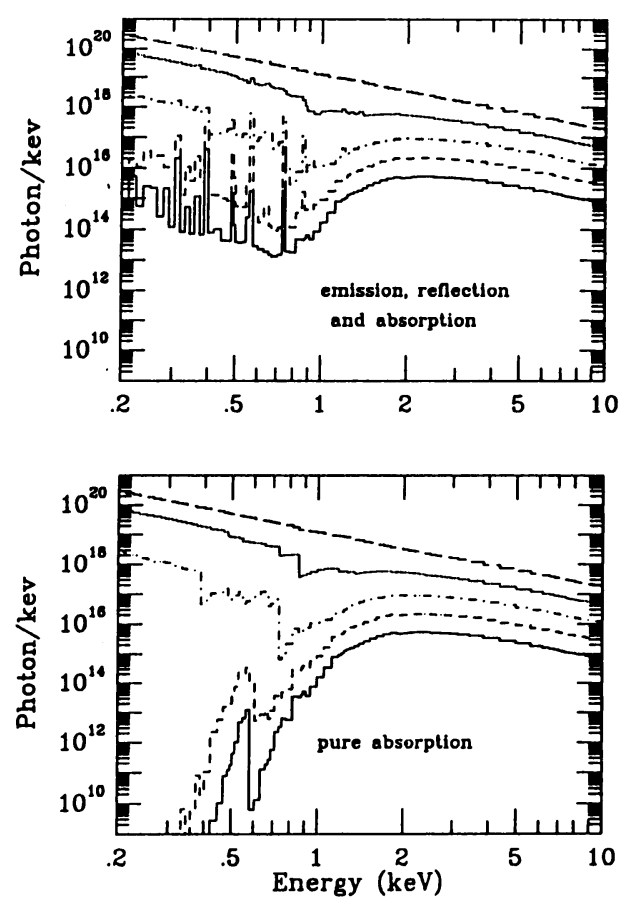

Fig. 1. Calculated X-ray spectra for an absorbing column of $10^{22.5} \mathrm{~cm}^{-2}$ and a range of ionization parameters (lowest curve is smallest ionization parameter). Top: Full spectrum (absorption, emission and reflection for a covering factor of 0.5 ). Bottom: The same but absorption only.

previous studies have neglected X-ray emission and low energy reflection by ionized gas. As shown by Netzer (1993), and below, this is not justified and can result in a wrong interpretation.

Typical reflection by moderate column density gas, at $0.5-3 \mathrm{keV}$, is of order $1 \%$ of the incident continuum flux for a covering factor of 0.5 . At lower energies, and for larger column density material (e.g. the surface of an accretion disk) it can be much larger, close to $100 \%$. This sets a limit to the depth of the observed X-ray absorption features (clouds on the line of sight) and gives the impression of a soft excess (the ionized accretion disk case)

Recent calculations that I have performed demonstrate this case. Fig. 1 shows an example of a $10^{22.5} \mathrm{~cm}^{-2}$ column density cloud, on the line of sight to a central $\mathrm{X}$-ray continuum source, for a range of possible ionization parameters. It demonstrates the very large difference between the pure absorption case (bottom part) and the more realistic case where emission and reflection are included. Clearly visible are emission lines and edges and the rising continuum, at low energies, resulting from $\mathrm{X}$-ray reflection (this effect was not discussed in detail by Lightman 

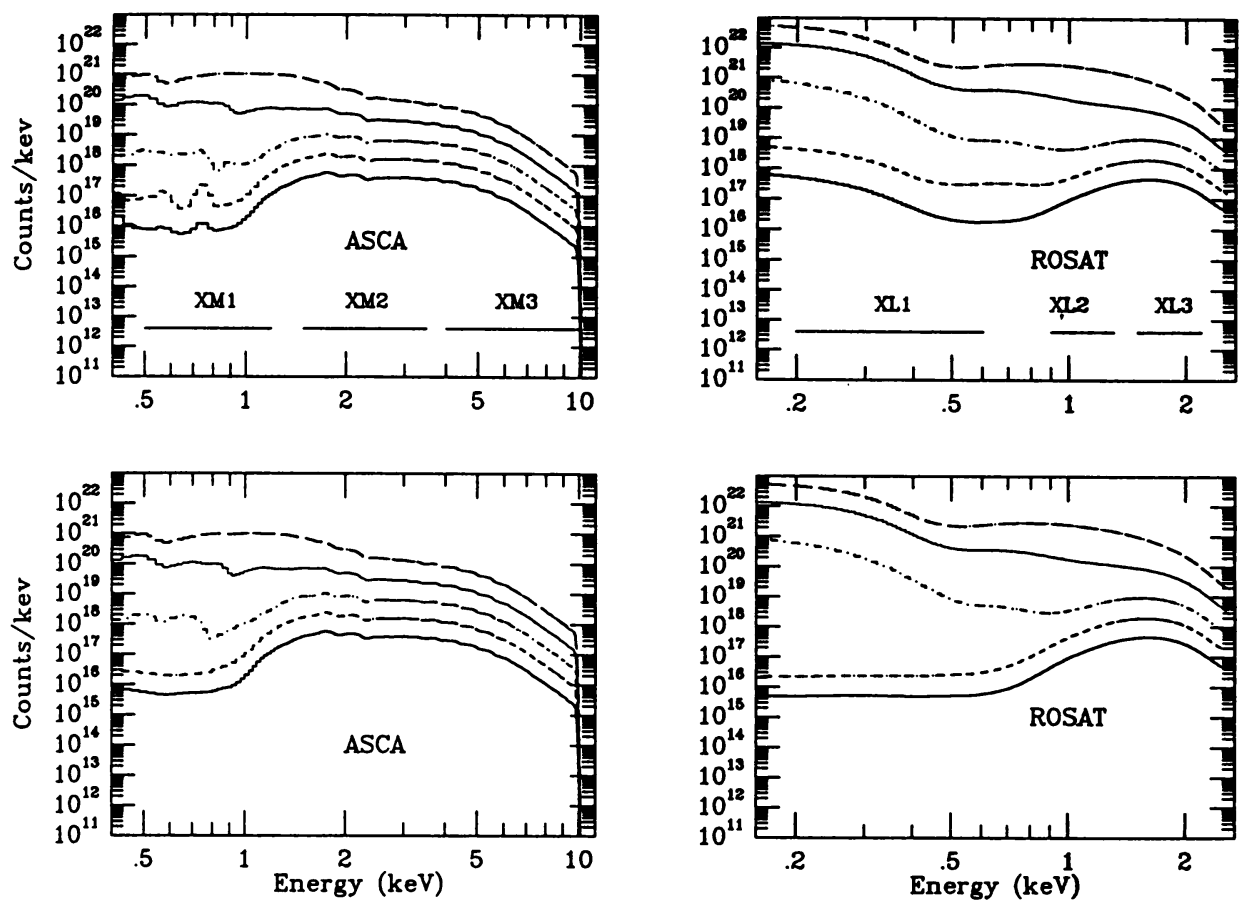

Fig. 2. The X-ray spectra of Fig. 1 as will be seen by ROSAT PSPC and ASCA SIS. Note that $A S C A$ has little capability in distinguishing pure absorption from the combined spectrum. ROSAT with its lower energy sensitivity, is much better in this respect. The $\mathrm{X}$-ray colors mentioned below are marked on the top panels.

and White, 1988, who emphasized reflection by neutral gas).

The appearance of observed X-ray spectra depend on the experiment resolution and sensitivity. Thus the ROSAT PSPC has very poor resolution, small energy range (0.1-2.5 $\mathrm{keV})$ and high sensitivity at low energies. This makes it difficuly to detect X-ray features. The recently launched $A S C A$, with its much improved resolution and range $(0.4-10 \mathrm{keV})$, is potentially better, but the limited sensitivity at low energies, and partial charge in the CCD detectors, make it difficult to distinguish pure absorption cases from others. This is illustrated in Fig. 2 that shows the predicted theoretical spectra after convolving with the response matrices of the above two experiments. The shape of the spectrum at low energies is enough to separate the two cases of Fig. 1, using ROSAT (PSPC), but not in the ASCA (SIS) case. 


\section{X-ray Colors}

The idea of broad band data, and their ratios (colors), extensively used in the analysis of optical and infrared observations, can be adopted in the X-ray range. This method suffers from the poor spectral resolution but has the advantage of improved $S / N$ and of uncovering the overall energy distribution of the source. Recently we (Netzer, Turner \& George, in praparation) have studied this idea by investigating several possible X-ray colors and their relations to the spectrum. Such bands must be specified for every experiment and our best choice for ROSAT and $A S C A$ are shown in figure 2. We find this method very useful in separating AGNs into groups according to their continuum properties. We also find that the $\mathrm{X}$-ray colors, combined with time variability data, can distinguish those AGNs with intrinsic continuum shape variations from those where the spectral changes are due to changes in the level of ionization of the absorbing material. We expect that the method will be very useful in analyzing the newly obtained $A S C A$ data.

\section{Acknowledgements}

It is a pleasure to acknowledge much help from members of the Laboratory for High Energy Astrophysics at Nasa/Goddard and a senior NRC research fellowship. The comments and contributions of my collaborators, Jane Turner and Ian George, helped a lot in preparing this paper.

\section{References}

Halpern, J.P, 1984, ApJ, 281, 90.

Krolik, J.H., and Kallamn, T.R., 1984, ApJ, 286, 366

Lightman, A.P., and White, T.R., 1988, ApJ, 335, 57

Netzer, H., 1993, ApJ, 411, 594.

Reichert, G.A., Mushotzky, R.F., Petre, R., and Holt, S.S, 1985, Ap.J. 296, 69.

Reichert, G.A., Mushotzky, R.F., and Holt, S.S, 1986, Ap.J. 303, 87.

Weaver, K.A., et al., 1993, ApJ (submitted)

Yaqoob, T., Warwick, R.S., and Pounds, K.A., 1989, MNRAS, 236, 153 